\title{
SUSLIN'S CONJECTURE ON THE REDUCED WHITEHEAD GROUP OF A SIMPLE ALGEBRA
}

\author{
ALEXANDER MERKURJEV
}

\begin{abstract}
In 1991, A. Suslin conjectured that if the index of a central simple algebra $A$ is not square-free, then the reduced Whitehead group of $A$ is nontrivial generically. We prove this conjecture in the present paper.
\end{abstract}

\section{INTRODUCTION}

Let $A$ be a central simple algebra over a field $F$. The reduced norm homomorphism $A^{\times} \rightarrow F^{\times}$yields a homomorphism

$$
\operatorname{Nrd}: K_{1}(A) \rightarrow F^{\times}=K_{1}(F) .
$$

The kernel $\mathrm{SK}_{1}(A)$ of $\mathrm{Nrd}$ is the reduced Whitehead group of $A$. Wang proved in $[25]$ that if $\operatorname{ind}(A)$ is a square-free integer, then $\operatorname{SK}_{1}(A)=0$. He also proved that the reduced Whitehead group is always trivial if $F$ is a number field. Platonov found examples of $A$ with nontrivial $\mathrm{SK}_{1}(A)$ (see [17]).

In 1991, Suslin conjectured in [23] that if ind $(A)$ is not square-free, then the reduced Whitehead group $\operatorname{SK}_{1}(A)$ of $A$ is generically nontrivial, i.e., there is a field extension $L / F$ such that $\operatorname{SK}_{1}\left(A \otimes_{F} L\right) \neq 0$.

Suslin's Conjecture was proved in the case when $\operatorname{ind}(A)$ is divisible by 4 (see [13] and [15]).

In this paper we prove Suslin's Conjecture (Theorem 8.1):

Theorem. Let $A$ be a central simple $F$-algebra. If ind $(A)$ is not square-free, then there is a field extension $L / F$ such that $\operatorname{SK}_{1}\left(A \otimes_{F} L\right) \neq 0$.

Note that the group $\operatorname{SK}_{1}(A)$ coincides with the group of $R$-equivalence classes in the special linear group $\mathbf{S L}_{1}(A)$. In particular, generic non-triviality of the reduced Whitehead group of $A$ implies that $\mathbf{S L}_{1}(A)$ is not a retract rational variety (Corollary 8.2).

\section{CyCle modules AND SPECTRAl SEQUenCES}

Let $Z$ be a variety over a field $F$ and let $M_{*}$ be a cycle module over $Z$ (see $[20, \S 2])$. This is a collection of group $M_{n}(z)$ for $n \in \mathbb{Z}$ and a point $z: L \rightarrow Z$

Date: November, 2014.

Key words and phrases. simple algebras; reduced Whitehead group; algebraic $K$-theory; algebraic cycles. 2010 Mathematical Subject Classifications: 19B99, 14C25 .

The work has been supported by the NSF grant DMS \#1160206 and the Guggenheim Fellowship. The author thanks the Max Planck Institute (Bonn) for hospitality. 
over $F$ having certain compatibility properties. We write $K_{*}$ for the cycle module over Spec $F$ given by (Quillen's) $K$-groups (see [20, Remark 2.5]).

For every integer $r \geq 0$, denote by $Z^{(r)}$ the set of points of $Z$ of codimension $r$. We write $A^{r}\left(Z, M_{n}\right)$ for the homology group of the complex [20, §5]

$$
\coprod_{z \in Z^{(r-1)}} M_{n-r+1} F(z) \stackrel{\partial}{\longrightarrow} \coprod_{z \in Z^{(r)}} M_{n-r} F(z) \stackrel{\partial}{\longrightarrow} \coprod_{z \in Z^{(r+1)}} M_{n-r-1} F(z) .
$$

For example, $A^{r}\left(Z, K_{r}\right)$ is the Chow group $\mathrm{CH}^{r}(Z)$ of classes of codimension $r$ algebraic cycles on $Z$. If $Z$ is smooth, $A^{*}\left(Z, K_{*}\right)$ is a bi-graded commutative ring.

If $f: Y \rightarrow Z$ is a flat morphism of equidimensional varieties and $M$ a cycle module over $Y$, for every $n \in \mathbb{Z}$, there is a spectral sequence [20, Corollary 8.2]

$$
E_{1}^{r, s}(f, n)=\coprod_{z \in Z^{(r)}} A^{s}\left(f^{-1}(z), M_{n-r}\right) \Rightarrow A^{r+s}\left(Y, M_{n}\right),
$$

where $f^{-1}(z)$ is the fiber of $f$ over $z \in Z$.

Very often we will be considering the projections $f: W \times Z \rightarrow Z$ with $Z$ and $W$ smooth varieties. In this case $f^{-1}(z)=W_{F(z)}$. The associated spectral sequences have the following functorial properties. A morphism $h: W \rightarrow W^{\prime}$ of smooth varieties yields a pull-back morphism of spectral sequences

$$
h^{*}: E_{*}^{*, *}\left(f^{\prime}, n\right) \rightarrow E_{*}^{*, *}(f, n)
$$

for every $n$ (here $f^{\prime}: W^{\prime} \times Z \rightarrow Z$ is the projection). If $h$ is a closed embedding of codimension $c$, we have a push-forward morphism of spectral sequences

$$
h_{*}: E_{*}^{*, *}(f, n) \rightarrow E_{*}^{*, *+c}\left(f^{\prime}, n+c\right) .
$$

More generally, every correspondence $\lambda$ between $W$ and $W^{\prime}$ of degree $d$ (see $[3, \S 63])$ yields a morphism

$$
\lambda^{*}: h^{*}: E_{*}^{*, *}\left(f^{\prime}, n\right) \rightarrow E_{*}^{*, *-d}(f, n-d) .
$$

This is because the four basic maps of complexes of $W \times Z$ and $W^{\prime} \times Z$ respect the filtration when projected to $Z$ (see $[20, \S 3]$ ).

\section{Chern Classes}

Let $X$ be a smooth variety. There are Chern classes (see [7]):

$$
c_{i, n}: K_{n}(X) \rightarrow A^{i-n}\left(X, K_{i}\right)
$$

for $i \geq n \geq 0$. We will only need the classes

$$
c_{i}:=c_{i+1,1}: K_{1}(X) \rightarrow A^{i}\left(X, K_{i+1}\right) .
$$

There is the following product formula (see [21]):

Proposition 3.1. If $x \in K_{0}(X)$ is the class of a line bundle $L$ and $y \in K_{1}(X)$, we have

$$
c_{i}(x y)=\sum_{j=0}^{i}(-1)^{j}\left(\begin{array}{l}
i \\
j
\end{array}\right) h^{j} \cup c_{i-j}(y)
$$


where $h$ is the first (classical) Chern class of $L$ in $A^{1}\left(X, K_{1}\right)=\mathrm{CH}^{1}(X)$.

Let $E \rightarrow X$ be a vector bundle of rank $n$ and $\mathbf{S L}(E)$ the group scheme over $Z$ of determinant 1 automorphisms of $E$. We will be using the following result due to Suslin [22, Th. 4.2].

Proposition 3.2. If $X$ is a smooth variety, the ring $A^{*}\left(\mathbf{S L}(E), K_{*}\right)$ is almost exterior algebra over $A^{*}\left(X, K_{*}\right)$ with generators $c_{1}(\beta), c_{2}(\beta), \ldots, c_{n-1}(\beta)$, where $\beta \in K_{1}(\mathbf{S L}(E))$ is the generic element. In particular,

$$
\mathrm{CH}(\mathbf{S L}(E)) \simeq \mathrm{CH}(X) .
$$

\section{Severi-Brauer varieties}

Let $A$ be a central simple algebra of degree $n$ over $F, X=\mathrm{SB}(A)$ the SeveriBrauer variety of rank $n$ right ideals of $A$. If $A$ is split, i.e., $A=\operatorname{End}(V)$ for a vector space of dimension $n$, the variety $X$ is isomorphic to the projective space $\mathbb{P}(V)$.

The variety $X$ has a point over a field extension $L / F$ if and only if $A$ is split over $L$, i.e., $A_{L}:=A \otimes_{F} L \simeq M_{n}(L)$.

Write $h$ for the class of a hyperplane section in $\mathrm{CH}^{1}\left(\mathbb{P}^{n-1}\right)$. The Chow group $\mathrm{CH}^{i}\left(\mathbb{P}^{n-1}\right)$ for $i=0,1, \ldots, n-1$ is infinite cyclic generated by $h^{i}$.

In the general case, the kernel of the degree homomorphism

$$
\operatorname{deg}: \mathrm{CH}^{i}(X) \rightarrow \mathrm{CH}^{i}\left(X_{\text {sep }}\right)=\mathbb{Z} h^{i}
$$

coincides with the torsion part of $\mathrm{CH}^{i}(X)$. The group $\mathrm{CH}_{0}(X)$ is torsion free (see [16] or [1, Corollary 7.3]). Therefore, the classes in $\mathrm{CH}_{0}(X)$ of every two points of the same degree are equal.

If $A=M_{m}(B)$ for a central simple algebra $B$ over $F$ and $S=\mathrm{SB}(B)$, then $S$ is a closed subvariety of $X=\mathrm{SB}(A)$. Moreover, the Chow motive $M(X)$ of $X$ is isomorphic to the direct sum $M(S) \oplus M(S)\{k\} \oplus \cdots \oplus M(S)\{(m-1) k\}$, where $k=n / m$.

Let $I \rightarrow X$ be the tautological rank $n$ vector bundle. The fiber of this bundle over a right ideal in $A$, a point of $X$, is the ideal itself. In the split case $A=\operatorname{End}(V)$, where $V$ is a vector space of dimension $n$, a line $l \subset V$ as a point of $X=\mathbb{P}(V)$ corresponds to the right ideal $\operatorname{Hom}(V, l)=V^{\vee} \otimes l$. Therefore, $I=V^{\vee} \otimes L_{t}$, where $L_{t}$ is the tautological line bundle over $\mathbb{P}(V)$. The canonical bundle $J$ over $X$, the dual of $I$, is equal then to $V \otimes L_{c}$, where $L_{c}$ is the canonical line bundle, dual of $L_{t}$. We have in the split case

$$
X \times X=X \times \mathbb{P}(V)=\mathbb{P}_{X}(V)=\mathbb{P}_{X}\left(V \otimes L_{c}\right)=\mathbb{P}_{X}(J) .
$$

Note that the projective linear group $\mathbf{P G L}(V)$ acts on $\mathbb{P}(V)$ and the vector bundles $I$ and $J$. In the general case, twisting by the $\mathbf{P G L}(V)$-torsor corresponding to the algebra $A$, we get an isomorphism

$$
X \times X \simeq \mathbb{P}_{X}(J),
$$

i.e., $X \times X$ is a projective vector bundle of $J$ over $X$ (with respect to the first of the two projections $\left.q_{1}, q_{2}: X \times X \rightarrow X\right)$. 
The tautological line bundle $\mathcal{L}_{t}$ over $X \times X=\mathbb{P}_{X}(J)$ is the sub-bundle $q_{1}^{*}\left(L_{t}\right) \otimes q_{2}^{*}\left(L_{c}\right)$ of the bundle $q_{1}^{*}(J)=V \otimes q_{2}^{*}\left(L_{c}\right)$ in the split case. Therefore,

$$
\mathcal{L}_{c}=q_{1}^{*}\left(L_{c}\right) \otimes q_{2}^{*}\left(L_{t}\right),
$$

where $\mathcal{L}_{c}$ is the canonical bundle over over $X \times X$.

Lemma 4.2. Let $x \in X$ be a closed point. Then the push-forward homomorphism $\mathbb{Z}=\mathrm{CH}\left(X_{F(x)}\right) \rightarrow \mathrm{CH}(X \times X)$ for the closed embedding

$$
i: X_{F(x)}=X \times \operatorname{Spec} F(x) \hookrightarrow X \times X
$$

depends only on the degree of $x$.

Proof. The canonical line bundle $L$ over the projective space is the pull-back of the canonical bundle $\mathcal{L}$ on $X \times X$. Hence the class $h_{1}=c_{1}(L)$ is equal to $i^{*}(h)$, where $h=c_{1}(\mathcal{L})$. By the projection formula,

$$
i_{*}\left(h_{1}^{i}\right)=i_{*}\left(i^{*}\left(h^{i}\right)\right)=i_{*}(1) \cdot h^{i}=\left[X_{F(x)}\right] \cdot h^{i} .
$$

The class of $X_{F(x)}$ in $\mathrm{CH}(X \times X)$ is the image of $[X] \times[x]$ under the exterior product map

$$
\mathrm{CH}(X) \otimes \mathrm{CH}_{0}(X) \rightarrow \mathrm{CH}(X \times X) .
$$

Finally, the class of $x$ in $\mathrm{CH}_{0}(X)$ depends only on the degree of $x$.

Choose a splitting field extension $L / F$ of the smallest degree ind $(A)$. We have $X_{L} \simeq \mathbb{P}_{L}^{n-1}$. Let $l_{i} \in \mathrm{CH}_{i}\left(X_{L}\right)$ be the class of a projective linear subspace of dimension $i$ and $e_{i}=e_{i}(A)$ the image of $l_{i}$ under the norm homomorphism

$$
N_{L / F}: \mathrm{CH}_{i}\left(X_{L}\right) \rightarrow \mathrm{CH}_{i}(X) \text {. }
$$

Then $e_{i}$ is independent of the choice of $L$. Indeed, choose a closed point $x \in X$ such that $F(x) \simeq L$. Then $e_{i}$ is the image of $l_{i}$ under the composition

$$
\mathrm{CH}_{i}\left(X_{L}\right)=\mathrm{CH}_{i}\left(X_{F(x)}\right) \rightarrow \mathrm{CH}_{i}(X \times X) \rightarrow \mathrm{CH}_{i}(X),
$$

where the last map is induced by the first projection. By Lemma 4.2, the composition does not depend on the choice of $x$.

The proof of Lemma 4.2 shows that for every closed point $x \in X$, we have

$$
N_{F(x) / F}\left(l_{i}\right)=\frac{\operatorname{deg}(x)}{\operatorname{ind}(A)} e_{i}(A) .
$$

Lemma 4.4. If $K / F$ is a finite extension, then

$$
N_{K / F}\left(e_{i}\left(A_{K}\right)\right)=\frac{[K: F] \operatorname{ind}\left(A_{K}\right)}{\operatorname{ind}(A)} e_{i}(A) .
$$

Proof. Let $L / K$ be a splitting field of $A_{K}$ of degree ind $\left(A_{K}\right)$. Choose an $L$ point $\operatorname{Spec}(L) \rightarrow X$. Let $\{x\}$ be the image of this morphism. We have by $(4.3)$

$$
\begin{aligned}
N_{K / F}\left(e_{i}\left(A_{K}\right)\right)=N_{L / F}\left(e_{i}\left(A_{L}\right)\right)=[L: F(x)] \cdot N_{F(x) / F}\left(e_{i}\left(A_{L}\right)\right) \\
=\frac{[L: F]}{\operatorname{ind}(A)} e_{i}(A)=\frac{[K: F] \operatorname{ind}\left(A_{K}\right)}{\operatorname{ind}(A)} e_{i}(A) .
\end{aligned}
$$


Proposition 4.6. Let $p$ be a prime integer and $A$ a central simple $F$-algebra of p-primary degree, $X=\mathrm{SB}(A)$ the Severi-Brauer variety of $A$. Then $\mathrm{CH}_{i}(X)=$ $\mathbb{Z} e_{i}$ for $i=0,1, \ldots, p-2$. In particular, these groups have no torsion.

Proof. If $D$ is a division algebra Brauer equivalent to $A$, the Severi-Brauer variety $Y=\mathrm{SB}(D)$ is a closed subscheme of $X$. The push-forward map $\mathrm{CH}_{i}(Y) \rightarrow \mathrm{CH}_{i}(X)$ is an isomorphism for $i \leq \operatorname{dim}(Y)$ taking $e_{i}(D)$ to $e_{i}(A)$. Thus, in the proof of the proposition it suffices to assume that $A$ is a division algebra.

We prove the proposition by induction on $\operatorname{ind}(A)$. The case $\operatorname{ind}(A)=p$ was considered in [12, Corollary 8.7.2]. A standard restriction-corestriction argument reduces the proof to the case when $F$ is a $p$-special field, i.e., the degree of every finite field extension of $F$ is a power of $p$.

Let $A$ be a central division algebra of $p$-primary degree $n$ and $L \subset A$ a maximal subfield (of degree $n$ over $F$ ). The torus $T=R_{L / F}\left(\mathbb{G}_{m}\right) / \mathbb{G}_{m}$ acts naturally on $X$ making $X$ a toric variety. Write $U$ for the open $T$-invariant orbit and $Z$ for $X \backslash U$. Thus, $U$ is a $T$-torsor over $\operatorname{Spec}(F)$.

Conversely, let $U$ be a $T$-torsor over $\operatorname{Spec}(F)$ and let $A$ be a central simple algebra degree $n$ over $F$ with class in the relative Brauer group $\operatorname{Br}(L / F)=$ $H^{1}(F, T)$ corresponding to the class of $U$. Then $U$ is the open orbit of the $T$-action on $\mathrm{SB}(A)$.

In the split case, $X=\mathbb{P}^{n-1}$ and $T$ is the torus of invertible diagonal matrices modulo the scalar matrices. Then $U$ consists of all points in $\mathbb{P}^{n-1}$ with all coordinates $\neq 0$. The $T$-orbits are the subsets in $\mathbb{P}^{n-1}$ with zeros on the fixed set of coordinates.

Let $\Sigma$ be the set of all $n$ primitive idempotents of $L \otimes_{F} F_{\text {sep }}=F_{\text {sep }} \times \cdots \times F_{\text {sep }}$. Every $\sigma \in \Sigma$ yields a co-character $\chi_{\sigma}: \mathbb{G}_{m, F_{\text {sep }}} \rightarrow T_{\text {sep }}$ which belongs to an edge (1-dimensional cone) in the fan of the toric variety $X_{\text {sep }}$. Moreover, the correspondence $\sigma \mapsto \chi_{\sigma}$ yields a bijection between the set of nonempty subsets in $\Sigma$ and the set of cones in the fan (or the set of $T$-orbits in $X_{\text {sep }}$ ). The absolute Galois group $\Gamma=\operatorname{Gal}\left(F_{\text {sep }} / F\right)$ of $F$ acts transitively on the set $\Sigma$.

Lemma 4.7. We have $\mathrm{CH}_{i}(U)=0$ for $i=0,1, \ldots, p-2$.

Proof. If $\operatorname{ind}(A)=p$, every cycle $c$ in $\mathrm{CH}_{i}(U)$ comes by restriction from $\mathrm{CH}_{i}(X)=p \mathbb{Z}$ and therefore, by the norm, comes from $\mathrm{CH}_{i}\left(X_{L}\right)$. Hence $c$ comes by the norm from $\mathrm{CH}_{i}\left(U_{L}\right)$. But $U_{L} \simeq T_{L}$, hence $\mathrm{CH}_{i}\left(U_{L}\right)=0$.

In the general case, since $F$ is a $p$-special field, there is a subfield $K \subset L$ of degree $p$ over $F$. Consider the subtorus $S:=R_{K / F}\left(\mathbb{G}_{m}\right) / \mathbb{G}_{m}$ of $T$, the $S$-torsor

$$
f: U \rightarrow X:=U / S
$$

and Rost's spectral sequence for $f$ converging to $\mathrm{CH}_{i}(U)$. On the zero diagonal, we have the groups $\coprod_{x \in X_{(j)}} \mathrm{CH}_{k}\left(f^{-1}(x)\right)$ with $j+k=i$. Note that $f^{-1}(x)$ is an $S$-torsor over Spec $F(x)$. Since $k \leq i \leq p-2$, by the first part of the proof, $\mathrm{CH}_{k}\left(f^{-1}(x)\right)=0$. 
The $T$-orbits in $Z_{\text {sep }}$ correspond to proper subsets of the set of $\Sigma$. No such subset is fixed by $\Gamma$, hence no orbit in $Z_{\text {sep }}$ is fixed by $\Gamma$.

We have a sequence of closed $T$-invariant subsets

$$
Z=Z_{0} \supset Z_{1} \supset \cdots \supset Z_{m} \supset Z_{m+1}=\emptyset
$$

such that every variety $\left(Z_{j} \backslash Z_{j+1}\right)_{\text {sep }}$ is the disjoint union of $T$-orbits of the same dimension which are permuted by $\Gamma$. It follows that each $Z_{j} \backslash Z_{j+1}$ is a disjoint union of varieties defined over finite separable field extensions $K / F$ corresponding to the stabilizers $\Gamma^{\prime} \subset \Gamma$ of $T$-orbits. The group $\Gamma^{\prime}$ does not act transitively on the set $\Sigma$, hence $L \otimes_{F} K$ is not a field and therefore, $A_{K}$ is not a division algebra, i.e., $\operatorname{ind}\left(A_{K}\right)<\operatorname{ind}(A)$.

If $W$ is a scheme over a finite separable field extension $K / F$, the norm map $\mathrm{CH}\left(W \otimes_{F} K\right) \rightarrow \mathrm{CH}(W)$ is surjective, since $K$ is a direct factor of $K \otimes_{F} K$.

Fix an integer $i=0,1, \ldots, p-2$. We say that a variety $W$ over $F$ satisfies the condition $(*)$ if $\mathrm{CH}_{i}(W)$ is generated by the images of the norm maps $\mathrm{CH}_{i}\left(W \otimes_{F} K\right) \rightarrow \mathrm{CH}_{i}(W)$ over finite field extensions $K / F$ with $\operatorname{ind}\left(A_{K}\right)<$ $\operatorname{ind}(A)$. We have proved that all the differences $Z_{j} \backslash Z_{j+1}$ satisfy $(*)$.

Let $W^{\prime}$ be a closed subvariety of $W$. The exactness of the localization sequence

$$
\mathrm{CH}_{i}\left(W^{\prime}\right) \rightarrow \mathrm{CH}_{i}(W) \rightarrow \mathrm{CH}_{i}\left(W \backslash W^{\prime}\right) \rightarrow 0
$$

shows that if $W^{\prime}$ and $W \backslash W^{\prime}$ satisfy $(*)$, then so does $W$. It follows from (4.8) that $Z$ satisfies $(*)$. By Lemma $4.7, U$ satisfies $(*)$, hence so does $X$.

By the induction hypothesis, $\mathrm{CH}_{i}\left(X_{K}\right)$ for $K$ as above, is generated by $e_{i}\left(A_{K}\right)$. By Lemma 4.4, $\mathrm{CH}_{i}(X)$ is generated by $e_{i}(A)$.

Corollary 4.9. The degree map $\mathrm{CH}_{i}(X) \rightarrow \mathrm{CH}_{i}\left(X_{\text {sep }}\right)=\mathbb{Z} l_{i}$ is injective, it takes $e_{i}$ to $\operatorname{ind}(A) l_{i}$. Thus, $\mathrm{CH}_{i}(X)$ is identified with the subgroup $\operatorname{ind}(A) \mathbb{Z} l_{i}$ in $\mathbb{Z} l_{i}$.

By the Projective Bundle Theorem, for every $j \geq 0$, we have

$$
\mathrm{CH}^{d-j}(X \times X) \simeq \mathrm{CH}^{d-j}(X) \oplus \mathrm{CH}^{d-j-1}(X) h \oplus \cdots \oplus \mathrm{CH}^{0}(X) h^{d-j},
$$

where $h \in \mathrm{CH}^{1}(X \times X)$ is the first Chern class of the canonical line bundle $\mathcal{L}_{c}$ over $X \times X$. The element $\lambda_{j}:=h^{d-j}$ can be viewed as a degree $j$ correspondence from $X$ to itself and hence $\lambda_{j}$ yields the homomorphism (see Section 2):

$$
\lambda_{j}^{*}: \mathrm{CH}_{0}(X) \rightarrow \mathrm{CH}_{j}(X) .
$$

Lemma 4.10. The maps $\lambda_{j}^{*}$ are isomorphisms for $j=0,1, \ldots, p-2$, taking $e_{0}(A)$ to $e_{j}(A)$.

Proof. By (4.1), in the split case, $h=h_{2}-h_{1}$, where $h_{i}$ are the pull-backs to $X \times X$ of the classes of the hyperplanes in $X$, hence $\lambda_{j}=\left(h_{2}-h_{1}\right)^{d-j}$. Therefore, $\lambda_{j}^{*}$ takes the generator $l_{0}$ of the infinite cyclic group $\mathrm{CH}_{0}(X)$ to the generator $l_{j}$ of $\mathrm{CH}_{j}(X)$.

By Proposition 4.6, in the general case, the degree map $\mathrm{CH}_{j}(X) \rightarrow \mathrm{CH}\left(X_{\mathrm{sep}}\right)=$ $\mathbb{Z} l_{j}$ identifies the group $\mathrm{CH}_{j}(X)$ with ind $(A) l_{j}$ by Corollary 4.9. The result follows. 


\section{Two CYCLE MODULES}

Let $A$ be a central simple algebra over $F$. The first cycle module $K_{*}^{Q A}$ is defined by

$$
K_{n}^{Q A}(L)=K_{n}\left(A_{L}\right)
$$

for a field extension $L / F$. The reduced norm map Nrd $: K_{n}\left(A_{L}\right) \rightarrow K_{n}(L)$ is defined for $n=0,1,2$ (see $[12, \S 6]$ ).

Let $G=\mathbf{S L}_{1}(A)$ be the algebraic group of reduced norm 1 elements in $A$. There is a canonical isomorphism (see [6, Proposition 7.3])

$$
A^{1}\left(G, K_{2}\right) \simeq \mathbb{Z}
$$

The group $A^{1}\left(G, K_{2}\right)$ does not change under field extensions.

In particular, we have a homomorphism

$$
\mathrm{Nrd}^{Q A}: A^{1}\left(G, K_{2}^{Q A}\right) \rightarrow A^{1}\left(G, K_{2}\right)=\mathbb{Z} .
$$

Let $X$ be the Severi-Brauer variety of $A$ of dimension $d$. We will be using another cycle module $K_{*}^{A}$ over $F$ defined by

$$
K_{n}^{A}(L)=A^{d}\left(X_{L}, K_{d+n}\right) .
$$

The push-forward homomorphism for the morphism $X_{L} \rightarrow \operatorname{Spec}(L)$ yields a map $A^{d}\left(X_{L}, K_{d+n}\right) \rightarrow K_{n}(L)$ and therefore, a morphism of cycle modules $K_{*}^{A} \rightarrow K_{*}$. In particular, we have a homomorphism

$$
\operatorname{Nrd}^{A}: A^{1}\left(G, K_{2}^{A}\right) \rightarrow A^{1}\left(G, K_{2}\right)=\mathbb{Z}
$$

There is a natural homomorphism $A^{d}\left(X_{L}, K_{d+n}\right) \rightarrow K_{n}\left(A_{L}\right)$ which is an isomorphism for $n=0$ and 1 (see [14]). Thus, we have a morphism of cycle modules $K_{*}^{A} \rightarrow K_{*}^{Q A}$ that is isomorphism in degree 0 and 1. It follows that the images of the maps $\mathrm{Nrd}^{Q A}$ and $\mathrm{Nrd}^{A}$ coincide.

If $A$ is split, $K_{*}^{Q A}=K_{*}^{A}=K_{*}$.

\section{A REDUCTION}

Recall that $G=\mathbf{S L}_{1}(A)$ for a central simple algebra $A$ of degree $n$ over $F$. For every commutative $F$-algebra $R$ there is a natural composition

$$
G(R) \hookrightarrow A_{R}^{\times} \rightarrow K_{1}\left(A_{R}\right),
$$

where $A_{R}=A \otimes_{F} R$.

Consider the generic point $\xi \in G(F[G])$ and its image $\xi_{F(G)}$ in $G(F(G))$. Let $\alpha$ be the image of $\xi$ under the map

$$
G(F[G]) \rightarrow K_{1}\left(A_{F[G]}\right),
$$

and let $\alpha_{F(G)}$ be the image of $\xi_{F(G)}$ under the map

$$
G(F(G)) \rightarrow K_{1}\left(A_{F(G)}\right) .
$$

We will prove that $\alpha_{F(G)}$ is nontrivial in $K_{1}\left(A_{F(G)}\right)$ when $A$ is a central simple algebra with ind $(A)$ not square-free. 
Filtering the category of coherent $A \otimes_{F} \mathcal{O}_{G}$-modules by codimension of support as in $[18, \S 7.5]$, we get the Brown-Gersten-Quillen spectral sequence (see $[18, \S 7])$

$$
E_{1}^{r, s}=\coprod_{g \in G^{(r)}} K_{-r-s}\left(A_{F(g)}\right) \Rightarrow K_{-r-s}\left(A_{F[G]}\right),
$$

where the limit is the $K$-group of the category of coherent $A \otimes_{F} \mathcal{O}_{G}$-modules equipped with the topological filtration (by codimension of support). In particular,

$$
E_{2}^{r, s}=A^{r}\left(G, K_{-s}^{Q A}\right)
$$

and the first term of the topological filtration on $K_{1}\left(A_{F[G]}\right)$ is equal to

$$
K_{1}\left(A_{F[G]}\right)^{(1)}=\operatorname{Ker}\left(K_{1}\left(A_{F[G]}\right) \rightarrow K_{1}\left(A_{F(G)}\right)\right) .
$$

The spectral sequence gives then a homomorphism

$$
\varepsilon: K_{1}\left(A_{F[G]}\right)^{(1)} \rightarrow A^{1}\left(G, K_{2}^{Q A}\right) .
$$

If $\alpha_{F(G)}$ is trivial in $K_{1}\left(A_{F(G)}\right)$, then $\alpha \in K_{1}\left(A_{F[G]}\right)^{(1)}$. Therefore, we have an element $\varepsilon(\alpha) \in A^{1}\left(G, K_{2}^{Q A}\right)$.

We compute $\varepsilon(\alpha)$ in the split case. We have $G=\mathbf{S L}_{n}$ and

$$
\alpha \in K_{1}\left(A_{F[G]}\right)=K_{1}(F[G])=K_{1}(G) .
$$

By [22, Th. 2.7], the first Chern class $c_{1}(\alpha)$ of $\alpha$ generates the group $A^{1}\left(G, K_{2}^{Q A}\right)=$ $A^{1}\left(G, K_{2}\right)=\mathbb{Z}$.

Lemma 6.1. In the split case, $\varepsilon(\alpha)=c_{1}(\alpha)$.

Proof. Let $H:=\mathbf{G L}_{n}$ and $\beta \in K_{1}(H)$ be the element given by the generic matrix. By [22, Th. 3.10], $\gamma_{i+1}(\beta) \in K_{1}(H)^{(i)}$ for all $i \geq 0$, where $\gamma$ is the gamma operation, and the image of $-\gamma_{2}(\beta)$ under the canonical homomorphism

$$
K_{1}(H)^{(1)} \rightarrow A^{1}\left(H, K_{2}\right)
$$

is equal to $c_{1}(\beta)$. On the other hand, the sum of $\gamma_{i}(\beta)$ for all $i \geq 1$ coincides with $\Lambda^{n}(\beta)=\operatorname{det}(\beta)$ by $\left[22\right.$, p. 65]. Hence $-\gamma_{2}(\beta) \equiv \beta-\operatorname{det}(\beta)$ modulo $K_{1}(H)^{(2)}$.

Pulling back with respect to the embedding of $G$ into $H$ we have $-\gamma_{2}(\alpha) \equiv \alpha$ modulo $K_{1}(G)^{(2)}$ since $\operatorname{det}(\alpha)$ is trivial and therefore, the image of $\alpha$ under the homomorphism $K_{1}(G)^{(1)} \rightarrow A^{1}\left(G, K_{2}\right)$ is equal to $c_{1}(\alpha)$.

Let $L / F$ be a splitting field of $A$. We have a commutative diagram

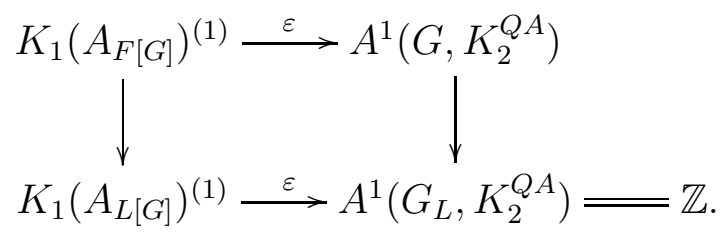

The right vertical homomorphism factors as follows:

$$
A^{1}\left(G, K_{2}^{Q A}\right) \stackrel{\mathrm{Nrd}^{Q A}}{\longrightarrow} A^{1}\left(G, K_{2}\right) \stackrel{\sim}{\rightarrow} A^{1}\left(G_{L}, K_{2}\right)=A^{1}\left(G_{L}, K_{2}^{Q A}\right) .
$$


Assume that $\alpha_{F(G)}$ is trivial in $K_{1}\left(A_{F(G)}\right)$, hence $\alpha \in K_{1}\left(A_{F[G]}\right)^{(1)}$. By Lemma 6.1, $\varepsilon(\alpha)_{L}$ in $A^{1}\left(G_{L}, K_{2}^{Q A}\right)=\mathbb{Z}$ is a generator. It follows that the image of $\varepsilon(\alpha)$ under the map $\operatorname{Nrd}^{Q A}: A^{1}\left(G, K_{2}^{Q A}\right) \rightarrow A^{1}\left(G, K_{2}\right)=\mathbb{Z}$ is equal to \pm 1 , hence $\mathrm{Nrd}^{Q A}$ is surjective.

We have proved:

Proposition 6.2. Suppose that the map $\operatorname{Nrd}^{Q A}: A^{1}\left(G, K_{2}^{Q A}\right) \rightarrow A^{1}\left(G, K_{2}\right)=$ $\mathbb{Z}$ is not surjective. Then Suslin's Conjecture holds for $A$.

Let $A$ be a central simple $F$-algebra such that ind $(A)$ is not square-free, i.e., $\operatorname{ind}(A)$ is divisible by $p^{2}$ for a prime integer $p$. We want to prove that $\operatorname{SK}_{1}(A)$ is nontrivial generically. Replacing $F$ by a field extension over which $A$ has index exactly $p^{2}$ and replacing $A$ by a Brauer equivalent division algebra, we may assume that $A$ is a division algebra of degree $p^{2}$. Moreover, an application of the index reduction formula shows that we may assume that $A$ is decomposable, i.e., $A$ is a tensor product of two algebras of degree $p$ (see [19, Theorem 1.20]).

We will prove that if $A$ is a decomposable division algebra of degree $p^{2}$, then the map $\operatorname{Nrd}^{Q A}$ is not surjective. Recall that the maps $\operatorname{Nrd}^{Q A}$ and $\operatorname{Nrd}^{A}$ have the same images. Therefore, it suffices to prove that the map $\mathrm{Nrd}^{A}$ : $A^{1}\left(G, K_{2}^{A}\right) \rightarrow A^{1}\left(G, K_{2}\right)=\mathbb{Z}$ is not surjective.

\section{A SPECTRAL SEQUENCE}

Let $A$ be a central simple $F$-algebra of degree $p^{2}$ and $X$ the Severi-Brauer variety of $A$ with $\operatorname{dim}(X)=d=p^{2}-1$. We would like to find a reasonable description the group $A^{1}\left(G, K_{2}^{A}\right)$ via algebraic cycles on $G \times X$.

Consider the spectral sequence associated with the projection $q: G \times X \rightarrow G$ (see Section 2):

$$
E_{1}^{r, s}=E_{1}^{r, s}(q, d+2)=\coprod_{g \in G^{(r)}} A^{s}\left(X_{F(g)}, K_{d+2-r}\right) \Rightarrow A^{r+s}\left(G \times X, K_{d+2}\right) .
$$

We have $E_{1}^{r, s}=0$ if $s>d$ and

$$
E_{2}^{r, d}=A^{r}\left(G, K_{2}^{A}\right) .
$$

There are no nontrivial differentials arriving at $E_{*}^{r, d}$.

Proposition 7.2. We have $E_{2}^{i, d+2-i}=0$ for $i=2,3, \ldots, p$. In particular,

$$
A^{1}\left(G, K_{2}^{A}\right)=E_{2}^{1, d}=E_{3}^{1, d}=\cdots=E_{p}^{1, d} .
$$

Proof. Let $j=i-2$ and $\lambda_{j}$ be the correspondence on $X \times X$ of degree $j$ considered in Section 4. By Lemma 4.10, the maps

$$
\lambda_{j}^{*}: \mathrm{CH}_{0}\left(X_{L}\right) \rightarrow \mathrm{CH}_{j}\left(X_{L}\right) .
$$

are isomorphisms for $j=0,1, \ldots, p-2$ and every field extension $L / F$. 
Consider the spectral sequence

$$
\widehat{E}_{1}^{r, s}:=E_{1}^{r, s}(q, d+i)=\coprod_{g \in G^{(r)}} A^{s}\left(X_{F(g)}, K_{d+i-r}\right) \Rightarrow A^{r+s}\left(G \times X, K_{d+i}\right) .
$$

The edge homomorphism

$$
\mathrm{CH}^{d+i}(G \times X)=A^{d+i}\left(G \times X, K_{d+i}\right) \rightarrow \widehat{E}_{2}^{i, d}
$$

is surjective. By Proposition 3.2,

$$
\mathrm{CH}^{d+i}(G \times X)=\mathrm{CH}^{d+i}(X)=0
$$

since $d+i>\operatorname{dim}(X)$. It follows that $\widehat{E}_{2}^{i, d}=0$.

The correspondence $\lambda_{j}$ yields a morphism between the spectral sequences (7.1) and (7.3). In particular, we have a homomorphism

$$
\tilde{\lambda}_{j}: \widehat{E}_{2}^{i, d} \rightarrow E_{2}^{i, d-i+2} .
$$

Since $\lambda_{j}^{*}$ is an isomorphism for $X_{L}$ for every field extension $L / F$, the map $\tilde{\lambda}_{j}$ is surjective. As $\widehat{E}_{2}^{i, d}=0$, we have $E_{2}^{i, d+2-i}=0$.

By Proposition 7.2, we have a differential

$$
A^{1}\left(G, K_{2}^{A}\right)=E_{p}^{1, d} \stackrel{\delta}{\longrightarrow} E_{p}^{p+1, d+1-p} .
$$

Proposition 7.4. If $\operatorname{ind}(A)=p$, the image of $\operatorname{Ker}(\delta)$ under the homomorphism

$$
\operatorname{Nrd}^{A}: A^{1}\left(G, K_{2}^{A}\right) \rightarrow A^{1}\left(G, K_{2}\right)=\mathbb{Z}
$$

is equal to $p \mathbb{Z}$.

We will prove this proposition in Section 10.

Let $A$ be a division algebra of degree $p^{2}$ over $F$. Choose a field extension $K / F$ such that $\operatorname{ind}\left(A_{K}\right)=p$ and set $\widetilde{A}=A_{K}, \widetilde{X}=X_{K}, \widetilde{G}=G_{K}$ and write $\widetilde{E}_{*}^{r, s}$ for the terms of the spectral sequence associated with the projection $\widetilde{G} \times \widetilde{X} \rightarrow \widetilde{G}$. We have the following commutative diagram

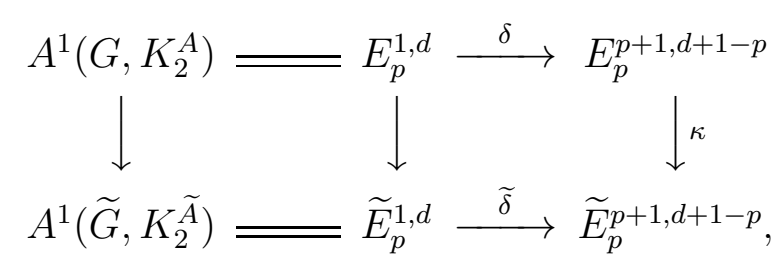

where $\delta$ and $\widetilde{\delta}$ are the differentials in the $p$-th pages of the spectral sequences.

Proposition 7.5. If $A$ is decomposable degree $p^{2}$ division algebra, then $\kappa$ : $E_{p}^{p+1, d+1-p} \rightarrow \widetilde{E}_{p}^{p+1, d+1-p}$ is the zero map.

We will prove this proposition in Section 11. 


\section{MAIN THEOREM}

We deduce the following theorem from Propositions 7.4 and 7.5.

Theorem 8.1. Let $A$ be a central simple $F$-algebra. If ind $(A)$ is not squarefree, then there is a field extension $L / F$ such that $\operatorname{SK}_{1}\left(A_{L}\right) \neq 0$.

Proof. We may assume that $A$ is a decomposable division algebra of degree $p^{2}$ for a prime integer $p$. Note that $\operatorname{ind}(\widetilde{A})=p$.

By Propositions 7.4 (applied to the algebra $\widetilde{A}$ ) and 7.5 , the image of the composition

$$
E_{p}^{1, d} \rightarrow \widetilde{E}_{p}^{1, d}=A^{1}\left(\widetilde{G}, K_{2}^{\widetilde{A}}\right) \stackrel{\operatorname{Nrd}^{\widetilde{A}}}{\longrightarrow} A^{1}\left(\widetilde{G}, K_{2}\right)=\mathbb{Z}
$$

is contained in $p \mathbb{Z}$. On the other hand, this composition coincides with

$$
E_{p}^{1, d}=A^{1}\left(G, K_{2}^{A}\right) \stackrel{\mathrm{Nrd}^{A}}{\longrightarrow} A^{1}\left(G, K_{2}\right)=\mathbb{Z} .
$$

Therefore, the norm homomorphism $\operatorname{Nrd}^{A}: A^{1}\left(G, K_{2}^{A}\right) \rightarrow A^{1}\left(G, K_{2}\right)$ is not surjective and this finishes the proof by Proposition 6.2 since $\operatorname{Im}\left(\mathrm{Nrd}^{Q A}\right)=$ $\operatorname{Im}\left(\mathrm{Nrd}^{A}\right)$.

An irreducible variety $Z$ over $F$ is called a retract rational variety if there exist rational morphisms $\alpha: Z \rightarrow \mathbb{P}^{m}$ and $\beta: \mathbb{P}^{m} \rightarrow Z$ for some $m$ such that the composition $\beta \circ \alpha$ is defined and equal to the identity of $Z$.

Corollary 8.2. Let $A$ be a central simple algebra over $F$. Then the following are equivalent:

(1) The group $\mathbf{S L}_{1}(A)$ is a retract rational variety;

(2) $\operatorname{SK}_{1}\left(A_{L}\right)=0$ for every field extension $L / F$;

(3) The index $\operatorname{ind}(A)$ is square-free.

Proof. (1) $\Rightarrow(2)$ : If $G:=\mathbf{S L}_{1}(A)$ is a retract rational variety, then $G_{L}$ is so for every field extension $L / F$. By [2, Proposition 11], the group of $R$-equivalence classes $G(L) / R$ is trivial. But $G(L) / R$ is isomorphic to $\operatorname{SK}_{1}\left(A_{L}\right)$ by $[24, \S 18.2]$.

$(2) \Rightarrow(1)$ : This is proved in [5, Proposition 2.4] and [10, Proposition 5.1].

$(2) \Leftrightarrow(3)$ : This is Theorem 8.1 .

\section{ChOw RING of $G$}

Let $G=\mathbf{S L}_{1}(A)$ for a central simple algebra $A$ of $p$-primary degree.

Lemma 9.1. The Chow groups $\mathrm{CH}^{i}(G)$ are trivial for $i=1,2, \ldots, p$ and $p \cdot \mathrm{CH}^{p+1}(G)=0$.

Proof. Since $\mathrm{CH}\left(G_{\text {sep }}\right)=\mathbb{Z}$ by $\left[22\right.$, Theorem 2.7], the groups $\mathrm{CH}^{i}(G)$ are $p$ primary torsion if $i>0$. As $K_{0}(G)=\mathbb{Z}$ (see [22, Theorem 4.1]), by [4, Example 15.3.6], we have $(i-1) ! \mathrm{CH}^{i}(G)=0$ for $i>0$. The result follows. 
Consider the Brown-Gersten-Quillen spectral sequence

$$
E_{2}^{r, s}=A^{r}\left(G, K_{-s}\right) \Rightarrow K_{-r-s}(G) .
$$

It follows from Lemma 9.1 that

$$
A^{1}\left(G, K_{2}\right)=E_{2}^{1,-2}=E_{3}^{1,-2}=\cdots=E_{p}^{1,-2} .
$$

Moreover, by $[8, \S 3]$,

$$
\mathrm{CH}^{p+1}(G)=E_{2}^{p+1,-p-1}=E_{3}^{p+1,-p-1}=\cdots=E_{p}^{p+1,-p-1} .
$$

We have then a differential

$$
\delta: A^{1}\left(G, K_{2}\right)=E_{p}^{1,-2} \rightarrow E_{p}^{p+1,-p-1}=\mathrm{CH}^{p+1}(G) .
$$

Write $h \in \mathrm{CH}^{p+1}(G)$ for the image under $\delta$ of the canonical generator of the group $A^{1}\left(G, K_{2}\right)=\mathbb{Z}$.

Proposition 9.2. Suppose that $\operatorname{ind}(A)=$, i.e., $A=M_{n}(B)$ and $G=$ $\mathbf{S L}_{n}(B)$ for some $n$ and a central division algebra $B$ of degree $p$. Then

$$
\mathrm{CH}^{*}(G)=\mathbb{Z} \oplus(\mathbb{Z} / p \mathbb{Z}) h \oplus(\mathbb{Z} / p \mathbb{Z}) h^{2} \oplus \cdots \oplus(\mathbb{Z} / p \mathbb{Z}) h^{p-1} .
$$

Proof. Induction on $n$. The case $n=1$ is done in [8, Theorem 9.7].

Let $H=\mathbf{S L}_{n-1}(B)$. We view $H$ as a subgroup of $G$ with respect to the embedding $x \mapsto \operatorname{diag}(1, x)$. Consider the closed subvariety $V$ of the affine space $B^{2 n}$ consisting of tuples $\left(b_{1}, \ldots, b_{n}, c_{1}, \ldots, c_{n}\right)$ such that $\sum b_{i} c_{i}=1$. Define the morphism

$$
f: G \rightarrow V, \quad a=\left(a_{i j}\right) \mapsto\left(a_{11}, \ldots, a_{1 n}, a_{11}^{\prime}, \ldots, a_{n 1}^{\prime}\right),
$$

where $\left(a_{i j}^{\prime}\right)=a^{-1}$. Clearly, $f$ is an $H$-torsor over $V$. For any field extension $L / F$, in the exact sequence of Galois cohomology

$$
G(L) \stackrel{f(L)}{\rightarrow} V(L) \rightarrow H^{1}(L, H) \stackrel{r}{\rightarrow} H^{1}(L, G)
$$

the map $r$ is a bijection (both sets are identified with $L^{\times} / \operatorname{Nrd}\left(B_{L}^{\times}\right)$and $r$ is the identity map by [11, Cor. 2.9.4]). Hence $f$ is surjective on $L$-points.

Let $W$ be the open subset of the affine space $B^{n}$ consisting of all tuples $\left(b_{1}, \ldots, b_{n}\right)$ such that $\sum b_{i} B=B$. We have $\mathrm{CH}^{i}(W)=0$ for $i>0$. The obvious projection $V \rightarrow W$ is an affine bundle, hence by the homotopy invariance property,

$$
\mathrm{CH}^{i}(V) \simeq \mathrm{CH}^{i}(W)=0
$$

for every $i>0$.

For every $m$, consider the spectral sequence associated with the morphism $f$ :

$$
E_{1}^{r, s}=E_{1}^{r, s}(f, m)=\coprod_{v \in V^{(r)}} A^{s}\left(f^{-1}(v), K_{m-r}\right) \Rightarrow A^{r+s}\left(G, K_{m}\right) .
$$

Since $f$ is surjective on $L$-points, $f^{-1}(v) \simeq H_{F(v)}$.

We claim that $E_{2}^{r, s}=0$ if $r+s=m$ and $r>0$. By induction, the group $A^{s}\left(G_{v}, K_{s}\right)=\mathrm{CH}^{s}\left(G_{v}\right)$ is trivial unless $s=(p+1) i$ for $i=0,1, \ldots, p-1$. In 
the latter case the map $\mathrm{CH}^{r}(V) \rightarrow E_{2}^{r, s}$ of multiplication by $h^{i}$ is surjective by the induction hypothesis. The claim follows from the triviality of $\mathrm{CH}^{r}(V)$ for $r>0$.

By the claim, $\mathrm{CH}(G) \simeq \mathrm{CH}\left(H_{F(V)}\right)$. The statement of the proposition follows by induction.

Corollary 9.4. Let $A$ be a central simple algebra of degree $p^{2}$. Then for every field extension $L / F$ such that $\operatorname{ind}\left(A_{L}\right) \leq p$, the map

$$
\mathrm{CH}(G) \rightarrow \mathrm{CH}\left(G_{L}\right)
$$

is surjective.

Proof. The element $h$ belongs to $\mathrm{CH}^{l+1}(G)$. As ind $\left(A_{L}\right) \leq p$, by Proposition 9.2 , the element $h_{L}$ generates the ring $\mathrm{CH}\left(G_{L}\right)$, whence the result.

\section{Proof of Proposition 7.4}

In this section, $A$ is a central simple algebra of degree $p^{2}$ and index $p$, so that $A=M_{p}(B)$, where $B$ is a division algebra of degree $p$. We write $S$ for the Severi-Brauer variety $\mathrm{SB}(B)$ of dimension $p-1$. Recall that the variety $S$ can be viewed as a closed subvariety of $X$. Moreover, the Chow motive $M(X)$ of $X$ is isomorphic to $M(S) \oplus M(S)\{p\} \oplus \cdots \oplus M(S)\{(p-1) p\}$.

Consider the spectral sequence associated with the projection $t: G \times S \rightarrow G$ :

$$
\hat{E}_{1}^{r, s}:=E_{1}^{r, s}(t, p+1)=\coprod_{g \in G^{(r)}} A^{s}\left(S_{F(g)}, K_{p+1-r}\right) \Rightarrow A^{p+q}\left(G \times S, K_{p+1}\right) .
$$

The embedding of $S$ into $X$ induces the push-forward morphisms between the spectral sequences (10.1) and (7.1). Moreover, (10.1) is a direct summand of (7.1). More precisely, the maps

$$
\hat{E}_{*}^{r, s} \rightarrow E_{*}^{r, s+d+1-p}
$$

are isomorphisms for $s=0,1, \ldots, p-1$.

By Proposition 7.2 , we have $E_{2}^{i, d+2-i}=0$ for $i=2,3, \ldots, p$. It follows that $\hat{E}_{2}^{i, p+1-i}=0$ for $i=2,3, \ldots, p$, i.e., all the terms but $\hat{E}_{2}^{p+1,0}$ on the diagonal $r+s=p+1$ on page $\hat{E}_{2}^{*, *}$ are zero. Moreover,

$$
E_{2}^{p+1, d+1-p}=\hat{E}_{2}^{p+1,0}=\mathrm{CH}^{p+1}(G) .
$$

It follows that

$$
A^{1}\left(G, K_{2}^{A}\right)=\hat{E}_{2}^{1, p-1}=\hat{E}_{3}^{1, p-1}=\cdots=\hat{E}_{p}^{1, p-1}
$$

and the only potentially nonzero differential starting in $\hat{E}_{\geq 2}^{1, p-1}$ appears on page $p$ :

$$
A^{1}\left(G, K_{2}^{A}\right)=\hat{E}_{p}^{1, p-1} \stackrel{\hat{\delta}}{\rightarrow} \hat{E}_{p}^{p+1,0} .
$$

The spectral sequence (10.1) yields then an exact sequence

$$
A^{l}\left(G \times S, K_{p+1}\right) \rightarrow \hat{E}_{p}^{1, p-1} \stackrel{\hat{\delta}}{\rightarrow} \hat{E}_{p}^{p+1,0} .
$$


The differential $\delta: E_{p}^{1, d} \rightarrow E_{p}^{p+1, d+1-p}$ in (10.1) is identified with the differential $\hat{\delta}: \hat{E}_{p}^{1, p-1} \rightarrow \hat{E}_{p}^{p+1,0}$ in (7.1). Thus, to prove the proposition, it suffices to show that the image of the composition

$$
A^{p}\left(G \times S, K_{p+1}\right) \rightarrow \hat{E}_{p}^{1, p-1}=A^{1}\left(G, K_{2}^{A}\right) \stackrel{\mathrm{Nrd}^{A}}{\longrightarrow} A^{1}\left(G, K_{2}\right)=\mathbb{Z}
$$

is equal to $p \mathbb{Z}$.

This composition is the push-forward homomorphism

$$
t_{*}: A^{p}\left(G \times S, K_{p+1}\right) \rightarrow A^{1}\left(G, K_{2}\right)=\mathbb{Z}
$$

with respect to the projection $t: G \times S \rightarrow G$.

Over $S$, the algebra $A$ is isomorphic to $\mathcal{E} n d_{\mathcal{O}_{S}}\left(J^{p}\right)$, where $J$ is the canonical vector bundle over $S$ of rank $p$. By Proposition 3.2,

$A^{p}\left(G \times S, K_{p+1}\right)=\mathrm{CH}^{p-1}(S) \cdot c_{1}(\beta) \oplus \mathrm{CH}^{p-2}(S) \cdot c_{2}(\beta) \oplus \cdots \oplus \mathrm{CH}^{0}(S) \cdot c_{p}(\beta)$, where $\beta \in K_{1}(G \times S)$ is the generic element.

Since the group $A^{1}\left(G, K_{2}\right)$ does not change under field extensions, it is sufficient to compute the image over a field extension $L / F$ splitting $A$. Over such a field extension the group $G_{L}$ is isomorphic to $\mathbf{S L}_{p^{2}}$. Let $\beta^{\prime} \in K_{1}\left(G_{L}\right)$ be the class of the generic matrix, so that $\beta=\left[L_{c}\right] \cdot t^{*}\left(\beta^{\prime}\right)$, where $L_{c}$ is the canonical line bundle over $X$. By Proposition 3.1,

$$
c_{i}(\beta)=\sum_{j=0}^{i}(-1)^{j}\left(\begin{array}{l}
i \\
j
\end{array}\right) h^{j} c_{i-j}\left(t^{*} \beta^{\prime}\right)
$$

for every $i=1,2, \ldots, p$, where $h \in \mathrm{CH}^{1}\left(S_{L}\right)$ is the first Chern class of $L_{c}$. Note that $c_{1}\left(\beta^{\prime}\right)$ is the canonical generator of $A^{1}\left(G_{L}, K_{2}\right)$. By the projection formula, the image of $t_{*}$ is the sum of the subgroups

$$
\left(\begin{array}{l}
i \\
j
\end{array}\right) \cdot t_{*}\left[\mathrm{CH}^{p-i}(S) \cdot h^{j}\right] \cdot c_{i-j}\left(\beta^{\prime}\right)
$$

over all $i=1,2, \ldots, p$ and $j=0,1, \ldots, i$. By dimension consideration, the subgroup is trivial if $j \neq i-1$. Consider the case $j=i-1$. If $p-i>0$, then the image of $\mathrm{CH}^{p-i}(S)$ in $\mathbb{Z}$ (when splitting $S$ ) is equal to $p \mathbb{Z}$. Finally, if $i=p$, the multiple $\left(\begin{array}{l}i \\ j\end{array}\right)$ is equal to $p$. The proposition is proved.

\section{Proof of Proposition 7.5}

In this section we assume that $A$ is a decomposable division algebra of degree $p^{2}$.

Since for every $i$ and $j$ with $i+j=d+2$ the natural homomorphism $E_{2}^{i, j} \rightarrow E_{p}^{i, j}$ is surjective, it is sufficient to prove that the homomorphism

$$
E_{2}^{p+1, d+1-p} \rightarrow \widetilde{E}_{2}^{p+1, d+1-p}
$$

is trivial. 
Let $L / F$ be a field extension. Considering $X$ over a separable closure of $L$ we get the homomorphisms

$$
A^{i}\left(X_{L}, K_{i+n}\right) \rightarrow A^{i}\left(X_{L_{\mathrm{sep}}}, K_{i+n}\right)=K_{n}(L)
$$

for $i=d+1-p$ and $n=0,1$. These homomorphisms induce the vertical maps in the following commutative diagram

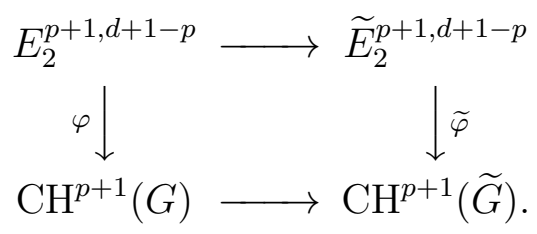

Since $\operatorname{ind}(\widetilde{A})=p$, it follows from (10.2) (applied to $\widetilde{A}$ ) that $\widetilde{\varphi}$ is an isomorphism. Thus, it is sufficient to prove that $\varphi=0$.

Recall that $A$ is a decomposable algebra. By a theorem of Karpenko [9, Th. $1]$,

$$
\operatorname{Im}\left(\mathrm{CH}^{d+1-p}\left(X_{F(g)}\right) \stackrel{\text { deg }}{\longrightarrow} \mathbb{Z}\right)= \begin{cases}p \mathbb{Z}, & \text { if ind } A_{F(g)}=p^{2} \\ \mathbb{Z}, & \text { if ind } A_{F(g)} \leq p\end{cases}
$$

Let $Y=\operatorname{SB}(p, A)$ be the generalized Severi-Brauer variety and set $m:=$ $\operatorname{dim}(Y)=p^{3}-p^{2}$. Consider the cycle module $M_{*}$ over $F$ defined by

$$
M_{n}(L)=A^{m}\left(Y_{L}, K_{n+m}\right) .
$$

There is the norm morphism $N: M_{*} \rightarrow K_{*}$ well defined. The variety $Y$ has a point over a field extension $L / F$ if and only if $\operatorname{ind}\left(A_{L}\right) \leq p$. It follows that

$$
\operatorname{Im}\left(A^{m}\left(Y_{L}, K_{m}\right) \stackrel{N}{\rightarrow} \mathbb{Z}\right)= \begin{cases}p \mathbb{Z}, & \text { if ind } A_{L}=p^{2} \\ \mathbb{Z}, & \text { if ind } A_{L} \leq p .\end{cases}
$$

Therefore the image of $\varphi$ coincide with the image of the map

$$
\psi: A^{p+1}\left(G, M_{p+1}\right) \rightarrow A^{p+1}\left(G, K_{p+1}\right)=\mathrm{CH}^{p+1}(G)
$$

induced by the norm map $N$. It is sufficient to prove that $\psi=0$.

The spectral sequence for the projection $G \times Y \rightarrow G$,

$$
E_{1}^{r, s}=\coprod_{g \in G^{(r)}} A^{s}\left(Y_{F(g)}, K_{m+p+1-r}\right) \Rightarrow A^{r+s}\left(G \times Y, K_{m+p+1}\right)
$$

yields a surjective homomorphism $\mathrm{CH}^{m+p+1}(G \times Y) \rightarrow A^{p+1}\left(G, M_{p+1}\right)$. The composition

$$
\mathrm{CH}^{m+p+1}(G \times Y) \rightarrow A^{p+1}\left(G, M_{p+1}\right) \stackrel{\psi}{\rightarrow} A^{p+1}\left(G, K_{p+1}\right)=\mathrm{CH}^{p+1}(G)
$$

is the push-forward homomorphism with respect to the projection $G \times Y \rightarrow G$. Thus, it is sufficient to show that the push-forward homomorphism

$$
\mathrm{CH}^{m+p+1}(G \times Y) \rightarrow \mathrm{CH}^{p+1}(G)
$$

is zero. 
Since ind $\left(A_{F(y)}\right) \leq p$ for every $y \in Y$, it follows from Corollary 9.4 that the map $\mathrm{CH}(G) \rightarrow \mathrm{CH}\left(G_{F(y)}\right)$ is surjective. Then the proof of [3, Lemma 88.5] yields the following lemma.

Lemma 11.2. The product homomorphism

$$
\mathrm{CH}(G) \otimes \mathrm{CH}(Y) \rightarrow \mathrm{CH}(G \times Y)
$$

is surjective.

Lemma 11.3. For every closed point $y \in Y$, the norm homomorphism

$$
N_{F(y)}: \mathrm{CH}^{p+1}\left(G_{F(y)}\right) \rightarrow \mathrm{CH}^{p+1}(G)
$$

is trivial.

Proof. The first map in the composition

$$
\mathrm{CH}^{p+1}(G) \rightarrow \mathrm{CH}^{p+1}\left(G_{F(y)}\right) \stackrel{N_{F(y) / F}}{\longrightarrow} \mathrm{CH}^{p+1}(G)
$$

is surjective by Corollary 9.4 since $\operatorname{ind}\left(A_{F(y)}\right) \leq p$. The composition is multiplication by $\operatorname{deg}(y)$. Note that $\operatorname{deg}(y)$ is divisible by $p \operatorname{since} \operatorname{ind}(A)=p^{2}$. The result follows from Lemma 9.1.

Proposition 11.4. If $A$ is a division algebra, the push-forward homomorphism

$$
\mathrm{CH}^{m+p+1}(G \times Y) \rightarrow \mathrm{CH}^{p+1}(G)
$$

is trivial.

Proof. By Lemma 11.2, it is sufficient to show that for every closed point $y \in Y$ the norm homomorphism $\mathrm{CH}^{p+1}\left(G_{F(y)}\right) \rightarrow \mathrm{CH}^{p+1}(G)$ is trivial. This is proved in Lemma 11.3.

\section{REFERENCES}

[1] V. Chernousov and A. Merkurjev, Connectedness of classes of fields and zero-cycles on projective homogeneous varieties, Compos. Math. 142 (2006), no. 6, 1522-1548.

[2] J-L. Colliot-Thélène and Jean-Jacques Sansuc, La R-équivalence sur les tores, Ann. Sci. École Norm. Sup. (4) 10 (1977), no. 2, 175-229.

[3] R. Elman, N. Karpenko, and Alexander Merkurjev, The algebraic and geometric theory of quadratic forms, American Mathematical Society Colloquium Publications, vol. 56, American Mathematical Society, Providence, RI, 2008.

[4] W. Fulton, Intersection theory, Springer-Verlag, Berlin, 1984.

[5] Ph. Gille, Le problème de Kneser-Tits, Astérisque (2009), no. 326, Exp. No. 983, vii, 39-81 (2010), Séminaire Bourbaki. Vol. 2007/2008.

[6] R. Garibaldi, A. Merkurjev, and Serre J.-P., Cohomological invariants in Galois cohomology, American Mathematical Society, Providence, RI, 2003.

[7] H. Gillet, Riemann-Roch theorems for higher algebraic K-theory, Adv. in Math. 40 (1981), no. 3, 203-289.

[8] N. Karpenko and A. Merkurjev, Motivic decomposition of compactifications of certain group varieties, arXiv:1402.5520 (2014).

[9] N. Karpenko, On topological filtration for Severi-Brauer varieties. II, 174 (1996), 45-48.

[10] N. Karpenko and A. Merkurjev, On standard norm varieties, Ann. Sci. Éc. Norm. Supér. (4) 46 (2013), no. 1, 175-214 (2013). 
[11] M.-A. Knus, A. Merkurjev, M. Rost, and J.-P. Tignol, The book of involutions, American Mathematical Society, Providence, RI, 1998, With a preface in French by J. Tits.

[12] A. S. Merkurjev and A. A. Suslin, K-cohomology of Severi-Brauer varieties and the norm residue homomorphism, Izv. Akad. Nauk SSSR Ser. Mat. 46 (1982), no. 5, 1011$1046,1135-1136$.

[13] A. S. Merkurjev, Generic element in $S K_{1}$ for simple algebras, K-Theory 7 (1993), no. $1,1-3$

[14] A. S. Merkurjev and A. A. Suslin, The group of $K_{1}$-zero-cycles on Severi-Brauer varieties, Nova J. Algebra Geom. 1 (1992), no. 3, 297-315.

[15] A. Merkurjev, The group $S K_{1}$ for simple algebras, $K$-Theory 37 (2006), no. 3, 311-319.

[16] I. Panin, Application of $K$-theory in algebraic geometry, Thesis, LOMI, Leningrad, 1984.

[17] V. Platonov, On the Tannaka-Artin problem, Dokl. Akad. Nauk SSSR 221 (1975), no. $5,1038-1041$.

[18] D. Quillen, Higher algebraic K-theory. I, (1973), 85-147. Lecture Notes in Math., Vol. 341.

[19] U. Reman, S. V. Tikhonov, and V. I. Yanchevskiı̌, Symbol algebras and the cyclicity of algebras after a scalar extension, Fundam. Prikl. Mat. 14 (2008), no. 6, 193-209.

[20] M. Rost, Chow groups with coefficients, Doc. Math. 1 (1996), No. 16, 319-393 (electronic).

[21] E. Shinder, On the motive of the group of units of a division algebra, J. K-Theory 13 (2014), no. 3, 533-561.

[22] A. A. Suslin, $K$-theory and $K$-cohomology of certain group varieties, in Algebraic $K$ theory, Advances in Soviet Mathematics, vol. 4, Providence, RI, American Mathematical Society, 1991, pp. 53-74.

[23] A. A. Suslin, $S K_{1}$ of division algebras and Galois cohomology, in Algebraic K-theory, Advances in Soviet Mathematics, vol. 4, Providence, RI, American Mathematical Society, 1991, pp. 75-99.

[24] V. E. Voskresenskiı̌, Algebraic groups and their birational invariants, Translations of Mathematical Monographs, vol. 179, American Mathematical Society, Providence, RI, 1998, Translated from the Russian manuscript by Boris Kunyavski [Boris È. Kunyavski1].

[25] S. Wang, On the commutator group of a simple algebra, Amer. J. Math. 72 (1950), 323-334.

Department of Mathematics, University of California, Los Angeles, CA 90095-1555, USA

E-mail address: merkurev@math.ucla.edu 